\title{
Linking Burnout to Psychological Well-being: The Mediating Role of Social Support and Learning Motivation
}

\author{
Abaid Ur Rehman ${ }^{1-3}$ \\ Tariq Mehmood Bhuttah ${ }^{4}$ \\ Xuqun You ${ }^{1,2}$ \\ 'School of Psychology, Shaanxi Normal \\ University, Xi'an, People's Republic of \\ China; ${ }^{2}$ Shaanxi Key Laboratory of \\ Behavior and Cognitive Neuroscience, \\ Shaanxi Normal University, Xi'an, \\ People's Republic of China; ${ }^{3}$ Department \\ of Psychology, BZU Bahadur Sub \\ Campus, Layyah, Pakistan; ${ }^{4}$ School of \\ Education, Shaanxi Normal University, \\ Xi'an, People's Republic of China
}

Correspondence: Xuqun You School of Psychology, Shaanxi Normal University, 199 South Chang'an Road, Yanta District, Xi'an 710062, People's Republic of China

Tel +86-29-85303852

Email youxuqun@snnu.edu.cn
This article was published in the following Dove Press journal: Psychology Research and Behavior Management

Background: University academic setting consists of specific life stressors such as burnout that influence a student's psychological well-being. Previous literature has shown the role of social support and learning motivation, but little research is known about how these variables, of social support and learning motivation, can mediate the relationship.

Methods: We recruited 486 participants from three Chinese universities with an age range of 18-35 years. Measures in the study include Maslach Burnout inventory (MBI) for college students, Multidimensional Scale of Perceived Social Support (MSPSS), motivation strategy learning scale, and psychological well-being by Ryff. Ethical approval was gained from the respondents.

Results: Findings suggest that social support plays a significant role in the link between burnout and subjective well-being. Indeed, the chain mediation model of social support and learning motivation significantly indicated the link between burnout and psychological wellbeing. These findings show that an increase in social support at an educational institute reduces the effects of burnout and enhances psychological well-being.

Conclusion: The study indicates a sound interpretation of psychological well-being and reducing the level of burnout. Subsequent research has found that social support and learning motivation could be an essential variable in calculating the educational success and learning motivation of the students.

Keywords: burnout, social support, learning motivation, psychological well-being

\section{Introduction}

In a daily social life, people contact each other. At some stage in life, they either help others or receive their help. Thus, to help each other in many ways could be considered as social support. To defeat the problem, the individual tries to find material or mystical support through this social networking. To smooth the progress of mind and individual happiness, the social support has a significant role in positive psychology. Different aspects, feelings of depersonalization, hopelessness, and emotional exhaustion have been referred to as the familiar terms of burnout, which arose during university life. Indeed, various studies showed that students experience burnout during their study period. ${ }^{1}$ However, subsequent research on social support and psychological well-being demonstrated that social support helps to reduce stress, sustain psychological condition at workplace. $^{2,3}$ In addition, motivation to learn has been considered important to the student learning process. This study intends to see the role of social support and learning motivation as a mediator in the link between burnout and psychological well-being. 


\section{Burnout and Psychological Well-Being}

Originally, burnout studies have been focused at workplace context, such as profession of human services. However, research on burnout has been linked to educational settings or academic situations. ${ }^{4}$ In the literature, burnout refers to the feeling of incompetence in terms of exhaustion and cynicism during student life. ${ }^{5}$ Burnout has been defined as a syndrome that consists of three factors; depersonalization, emotional exhaustion, and reduced personal accomplishment. ${ }^{6}$ Burnout is linked to main personal dysfunctions, for example, exhaustion, increased drug use, and insomnia. Burnout symptoms include social conflicts, lower efficiency, lower motivation, and dissatisfaction with work, and increased risk of health-related impairments. ${ }^{7}$ Several studies indicated that students experience burnout during their study period. ${ }^{1}$ Furthermore, studies also pointed out negative consequences such as mental health problems and discomfort caused by student stress in academic work. ${ }^{8}$ Research literature indicates that university students represent the future of their families and community. They face stressors in attaining their academic goals and have more demands and burdens. ${ }^{9}$ Therefore, these characteristics underscore the significance of psychological well-being of students. ${ }^{10}$

\section{Burnout, Social Support and Psychological Well-Being}

One of the most vigorous elements of study in modern times has a social support. Social support help to interact with family, friends, class fellows and many other people through different sides, for instance, instrumental, informative and emotional social support. Social support refers to the experience of being valued, nurtured, and respected by those who exist closest to the individual that may receive support from various sources, such as family, peers, friends, teachers, communities or one's an affiliated social group. To the extent that stress is an important component of burnout, this research is relevant to the development of a model of burnout among college students. ${ }^{6}$ Previous researchers have indicated stress as a predictor of social support among nursing students and act as a buffering among adolescents. For instance, an individual with increased social support under stressful situations shows better psychological well-being as compared to those who have less social support.

Another study also indicates that the mediating effect of social support identified that life stress exerted a significant effect on social support. ${ }^{11}$ Consequently, social support mediates the link between life stress and psychological outcome, such as stress and anger. For example, individuals are forced to gain support from their group members who are known to their problematic circumstances, they may show unfaithfulness and resentment and available support may be compromised. ${ }^{11}$ Moreover, individuals who perceive they are more blameworthy for their problematic issues may receive less support from others. ${ }^{12}$ Previous studies indicated that social interaction and social ties have been linked to better mental health and lower psychological stress. ${ }^{13}$ Moreover, asimilar study also suggests that better social interaction may positively relate to a lower level of mental illness. ${ }^{14}$ Literature lacks the role of social support in the context of burnout and psychological well-being, yet similar studies shows social support as a protective factor that mitigate the effect of stress on one's physical and psychological health. ${ }^{15}$ Studies also indicate that burnout may be contagious and perpetuate through social networks. ${ }^{16}$ Extensive literature suggests a strong link between perceived social support and student's well-being, such as social support reduces level of stress and depression among youth. ${ }^{17}$ Therefore, the mediating role of social support has become more significant in recent times. ${ }^{18}$

Past studies suggested that lack of social support leads to students' mental health problems. In an educational setting, pressure of higher education may become a person's extra emphatic to burnout. Students who represent low emotional relatedness to others or may lack social connections with others may interact with both physical as well as mental issues. ${ }^{19}$ Moreover, learners attachment to their families that plays a major role in supporting the learners' decisions to prioritize their learning. ${ }^{20}$ Indeed, literature and empirical research has indicated a link between social support and students' psychological problems. Therefore, the study tries to understand the role of social support in understanding students' psychological problems from the following aspects. First, social support may be very important to the individual's life. Inadequate social support has been linked with many psychological issues, such as loneliness, depression, and anxiety. ${ }^{21}$ Second, social support may be reviewed against life stress as a buffer, as well as health and wellness mediators for promoting physical and mental health. Research has also found that social support helps in protecting an individual's life crisis, for example, major stress and bereavement, which moderates these stressors linked to psychological well-being. ${ }^{22}$ Lastly, after reviewing the literature it may assumed that social support may protect 
and moderate the effects of stressors such as burnout on psychological wellbeing in one's life. Therefore, the current study will attempt to understand social support as a mediator in the link between burnout and psychological well-being.

\section{Burnout, Learning Motivation, and Psychological Well-Being}

Students' motivation to use the theory of convergence of the development within the individual has been determined by factors originating from within and factors that come from outside oneself. Motivation considered an internal process that is one of the main factors that determines the success rate of student learning. ${ }^{23}$ Motivation has been important in determining how many students will learn from a learning activity or how much they absorb the information presented to them. Students who are motivated to learn something will use higher cognitive processes in learning the material, so that they will absorb the material better.

Motivation for learning has been considered an important factor in education. Motivation refers a force that leads a student to perform academic responsibilities and better learning processes. If knowledge is considered being selfconstructed, referred by learning theory by constructivist, learning motivation has been assumed the crucial factor for task accomplishment. Some researchers have distinguished motivation in terms of intrinsic and extrinsic. ${ }^{24}$ While distinguishing these two terms, intrinsic motivation refers to a learning of something because of enjoyment and interest in learning, while extrinsic learning motivation refers to learning something because of certain ends, for example, to get a better place, to achieve a higher position in the class. ${ }^{25}$ In the literature, motivation has been considered as an influencing factor for students learning at university level. Students that were more intrinsically or autonomously motivated performed and showed more significant outcomes in learning, for example, better adjustment to institute, reduced stress level, and high in persistence. ${ }^{26}$ According to the theory of self-determination, autonomy in human beings is said to be a fundamental psychological need. ${ }^{24}$ Burnout was also associated with a higher dropout from university or college. ${ }^{27}$ Burnout among students also leads to low motivation, a high rate fn absenteeism, higher dropout percentage, and adverse academic performance. ${ }^{28}$

Indeed, self-determination theory postulates that motivation falls into the continuum that behaviors autonomously reflect motivation. Recent research provides motivation as a critical factor for the psychological well-being of an individual. Self-determined motivation among Chinese adolescents positively predicts academic success and better life satisfaction. ${ }^{29}$ In addition, the motivation has positive implications for well-being in both individual as well as collectivistic culture. ${ }^{30}$ Moreover, motivational orientation also influences an individual's adaptation to university, lowers the stress and mental health problems. ${ }^{31}$ Therefore, the current study sought to determine the link of burnout, learning motivation, and psychological well-being. In view of the previous literature, we concluded that learning motivation would mediate the link between burnout and psychological well-being. This indicates that the literature has not explored learning motivation as a mediator variable in the context of burnout and psychological well-being.

\section{The Present Study}

The aim of the current study is to verify the mediating role of social support as a social network mediation model and learning motivation concerning the mechanism of burnout linked to psychological well-being. First, our study could be noteworthy as it uses a sample of 486 Chinese university students with an age range of 18-35 years who may face burnout and consequently have low psychological wellbeing. It will uncover the link between burnout and psychological well-being and students may get help to understand and improve it. Second, university academic setting consists of specific life stressors; therefore, social support and students' relationships play a crucial role. Research also supports that social support as a buffering effect in the link between stressors and strains of burnout. ${ }^{32}$ Literature on cross-cultural psychology suggests various models, such as social networks or related to social support context that could mediate the link between burnout and psychological wellbeing. Therefore, social support not only helps to reduce life exhaustion but also increases mental health of students, such as psychological well-being. Third, past research lacks regarding social support and learning motivation as mediators as little research has been conducted in the context of burnout and psychological well-being. Fourth, the use of a chain mediation model could help to understand the combined effect of both social support and learning motivation in the link between burnout and psychological well-being. Fifth, the mediating model may also examine the independent effect of social support and learning motivation in this study, as this will help to determine which factor plays a more important role. 
In sum, four specific hypotheses are proposed based on the previous studies as follows: (1) burnout will be significantly linked to psychological well-being; (2) social support will mediate the association between burnout and psychological well-being; (3) learning motivation will mediate the link between burnout and psychological well-being; and (4) social support-learning motivation will mediated burnoutpsychological relationship through the chain mediation model. The hypothesized model is given below.

\section{Methods}

\section{Sample and Procedure}

About 486 university students (286 males and 200 females completed the study survey. Participants were between the ages of 18 and 35 years with mean age of 26.5 years. All the participants were from three Chinese Universities (Shaanxi Normal University, Xian International Studies, and Xian Jiaotong University and medical college). Students from the graduate school participated in the study to capture the experience of academic as well as university life exposure.

All the participants were enrolled at institutes at respective universities. Participant's demographic information (age, gender, and education level) and all questionnaires (burnout, students motivation, social support and psychological well-being scale) were used to collect the data.

\section{Ethical Approval and Informed Consent}

Ethical approval was obtained prior to start the research. Written informed consent forms wereprovided to all participants along with the main questionnaires. Participants were briefed about the research, and questionnaires were handed over to them. They were also informed that if they did not want to continue the survey, they could withdraw at any time without any penalty. All the respondents understood the study purpose and participated with written informed consent. The study received approval from the Shaanxi Normal University Ethical Committee (project ID: 09YJCXLX014).

\section{Instrument}

\section{Burnout Scale}

To measure Burnout, a modified scale of Maslach Burnout inventory (MBI) for college students was used. ${ }^{5}$ This modified version can be expected to assess the high possibility of burnout among college students during their studies. MBI has five-point rating scale that ranges from completely agree to disagree completely. The Maslach Burnout inventory uses a 15-item scale including three subcategories; emotional exhaustion, consisting of five items; cynicism with four items; and diminished efficacy with six times. Previous studies have reported internal consistency and test-retest for MBI for student's version. Alpha coefficients for emotional exhaustion, cynicism, and diminished efficacy respectively 88,90 , and $84 .{ }^{33}$ Results of the scale indicate that the more intrinsically a person is involved the chances of burnout would be low. ${ }^{34}$ The burnout inventory has been widely used in various disciplines. ${ }^{35}$

\section{Learning Motivation Scale}

Learning motivation was measured using a motivation strategy learning questionnaire (MSLQ). ${ }^{36}$ Several measures are used to evaluate academic motivation from the motivating part of the questionnaire of motivation strategy for learning: intrinsic motivation (4), external motivation (4), self-efficacy for learning and performance (8), task value motivation (6), learning belief motivation control (4), and test anxiety motivation (5). Learning motivation scale has been used in various empirical studies on different population samples to address both applied and theoretical purposes. The entire questionnaire or its subscales of learning motivation scale has been used. ${ }^{37,40}$ Alpha coefficients from manual of the scale have been noted as inherent motivation scale $0.74,0.62$ for the external, and the task value scale is 0.90 , ie the control level for learning beliefs, and 0.80 indicates that the test anxiety scale.

\section{Psychological Well-being Scale}

Eighteen items by Ryff and Keyes, ${ }^{41}$ based on a seven-point rating response scale was used to measure the construct of psychological well-being. It was measured using an anxiety stress scale. ${ }^{42}$ The subscales of psychological well-being have been illustrated in six domains: environmental mastery, positive relationship with others, autonomy, self-acceptance, personal growth, and purpose in life. ${ }^{43}$ The scale measures somatic tension and general fatigue related to one's feelings and stress-related physical symptoms. All items were summed, and scores ranged from 10 to 50, a low score indicates a low level of well-being and a high score reflects high psychological well-being. Eight items were reverse scored. The reliability of psychological well-being for the present sample has been reported as 0.83 . Due to the multidimensionality of psychological well-being questioned, 
we used psychological well-being as a global score in this study as it has been used in previous studies. ${ }^{44,45}$

\section{Social Support Scale}

Multidimensional Scale of Perceived Social Support (MSPSS) by Zimet et al, with 12-items, was used to measure social support. ${ }^{46}$ The scale measures social support from their perspectives: family, friends and significant others. Participants rate the scale based on a seven-point rating scale. The scale ranges from very strongly agree to very strongly disagree and a higher score predicts greater perceived social support, and low social support describes a low level of social support. Alpha coefficients and validity of the scale in previous research were found to be good. ${ }^{47} \mathrm{~A}$ reliability coefficient for family, friends and significant other were $0.82,0.86$, and 0.86 respectively.

\section{Statistical Analysis}

The IBM SPSS v24.0 (IBM Corporation, Armonk, NY, USA) was used to analyze the data. Prior to testing the model, we verified the necessary requisites to be compiled for the test. Among 500 respondents, missing values were $2.8 \%$. About $97.2 \%$ of the respondents (486 out of 500 ) had completed the questionnaires. A step-wise process was utilized to delete missing values list-wise, by leaving only the complete set of cases. ${ }^{48}$ All categorical variables such as age, gender and level of education were described as numbers and percentages. Descriptive statistics were also calculated to see the sociodemographic variables. Pearson correlations was used to measure the relationship between burnout, social support, learning motivation and psychological well-being. We applied IBM Amos 21 to test the mediation model. The mediation analysis was performed through Hayes process model and bootstrap processes using $95 \% \mathrm{CI}$ bias-corrected to analyze the mediating role of the variables as significant. In the analysis, burnout as an independent variable (X); social support and learning motivation as mediating variable $(\mathrm{M})$ variables; and psychological well-being as dependent variable (Y). The Hayes process analysis allows us to test the direct, total and indirect effect. The effect is significant if $95 \% \mathrm{CI}$ does not include 0 value. According to Fang et al, the $95 \%$ biased corrected bootstrap method was more suitable for non-normal data and that is statistically more power as compared to various other methods of analysis while testing the mediational effects. ${ }^{49}$

The following fit criteria were used assess the model as described by $\mathrm{Hu}$ and Bentler. ${ }^{50}$ The Comparative Fit Index
(CFI) $\geq 0.90$ recommended for acceptable fit criteria. ${ }^{51}$ The root mean square error of approximation (RMSEA) $<0.06$, suggests the basic model fit to the data. ${ }^{50}$ The TuckerLewis Index (TLI) $\geq 0.95$ recommended fit for hypothesized model and the standardized root-mean-square residual (SRMR) $<0.08$ indicating good fit. Finally, the study path diagrams show the hypothesized model for the study variables.

\section{Results}

\section{Demographic Characters of Study Sample}

Table 1 presents the demographic characteristics of the respondents. Among 486 respondents, the majority of the sample comprised of men $(n=286 ; 58.8 \%)$. In terms of age, most of the respondents were between the age of 20 and 25 years $(n=336 ; 69.1 \%)$, while only respondents 31 to 35 years of age were $(n=18 ; 3.7 \%)$. Regarding level of education, most of the respondents were enrolled in undergraduate programs $(n=325 ; 66.9 \%)$ and the lowest ratio were of postgraduate students $(\mathrm{n}=19 ; 3.9 \%)$

\section{Descriptive, Alpha Coefficients and Correlation Among Study Variables}

Table 2 describes the correlations between all study variables. Alpha coefficients presented in the table are satisfactory. Pearson correlation was computed to see the association among the study variables (burnout, learning motivation, social support, and psychological well-being. Significant positive correlation of all study variables was found.

Table I Demographic Characteristics of the Respondents $(n=486)$

\begin{tabular}{|l|l|}
\hline Characteristics & $\mathbf{n}(\%)$ \\
\hline Sex & \\
Men & $286(58.8)$ \\
Woman & $200(41.2)$ \\
\hline Age & \\
$20-25$ years & $336(69.1)$ \\
$26-30$ years & $132(27.2)$ \\
$31-35$ years & $18(3.7)$ \\
\hline Education & \\
Undergraduate & $325(66.9)$ \\
Graduate & $142(29.2)$ \\
Postgraduate & $19(3.9)$ \\
\hline
\end{tabular}


Table 2 Means, Standard Deviations (SD), Alpha Coefficient and Intercorrelations Among the Study Measures

\begin{tabular}{|l|l|l|l|l|l|l|}
\hline Variables & Mean & SD & $\boldsymbol{\alpha}$ & $\mathbf{I}$ & $\mathbf{2}$ & $\mathbf{3}$ \\
\hline I. Burnout & 47.16 & 27.26 & 0.91 & & & \\
2. Psychological & 69.29 & 37.38 & 0.80 & $0.25^{\mathrm{a}}$ & & \\
well-being & & & & & & \\
3. Social support & 43.72 & 25.77 & 0.86 & $0.39^{\mathrm{a}}$ & $0.55^{\mathrm{a}}$ &. \\
4. Learning motivation & 106.71 & 63.26 & 0.85 & $0.17^{\mathrm{a}}$ & $0.57^{\mathrm{a}}$ & $0.52^{\mathrm{a}}$ \\
\hline
\end{tabular}

Notes: $\alpha$ : referred to as Cronbach's alpha. ${ }^{a}$ Correlation is significant at the level 0.01 (two-tailed).

\section{Measurement Model}

In this study, we tested the measurement model based on four latent factors including burnout, social support, learning motivation, and psychological well-being with eight observed variables. Results show that all factor loading and study indicators for the latent factors at significant $p<0.001$ level. The measurement model suggests that latent factors with their respective indicators were well represented and indicated a good fit to the data. ${ }^{52}$ In the study model, the variable could correlate with all the measurement variables.

\section{Mediating Analysis}

At first, we tested the model 1 to examine social support and learning motivation as mediator in the association between academic burnout and psychological well-being and the data was analyzed. Findings showed the data: $\chi^{2}=88.91 .83 \mathrm{df}=27$, $p<0.001, \mathrm{RMSEA}=0.069, \mathrm{CFI}=0.98, \mathrm{TLI}=0.97$ which was satisfactory. In Figure 1, the mediation model indicated social support and learning motivation as mediator in the link between burnout and psychological well-being.

To find an optimal model, we tested the chain mediation in model 2. For this, we added the path from social support to learning motivation. Indexes of model fit seems to be better $\chi^{2}=78.18 \quad \mathrm{df}=28, \quad p<0.001, \quad$ RMSEA $\quad(90 \% \mathrm{CI})=0.063$, $\mathrm{CFI}=0.98, \mathrm{TLI}=0.97$. The model 2 consists of two mediators (social support, burnout, and psychological well-being) that fit the data. It is important to know that the relationship between burnout and psychological well-being is regulated by social support $(95 \% \mathrm{CI}=0.306,0.467)$ and learning motivation $(95 \% \mathrm{CI}=0.116,0.243)$. Therefore, we accept support for multiple intermediaries. In addition to this, a multiple mediator analysis was performed by comparing specific indirect effects to determine which factors have important mediating effects. The process of the bootstrap (in this study, we randomly selected 5000 bootstrap samples) was used to test the mediating effect. The importance of this can produce the most accurate indirect impact confidence interval. ${ }^{53}$

Table 3 shows the indirect effects in the final model (model 2) and the associated 95\% confidence intervals. As expected, independent mediation of social support $(95 \%$

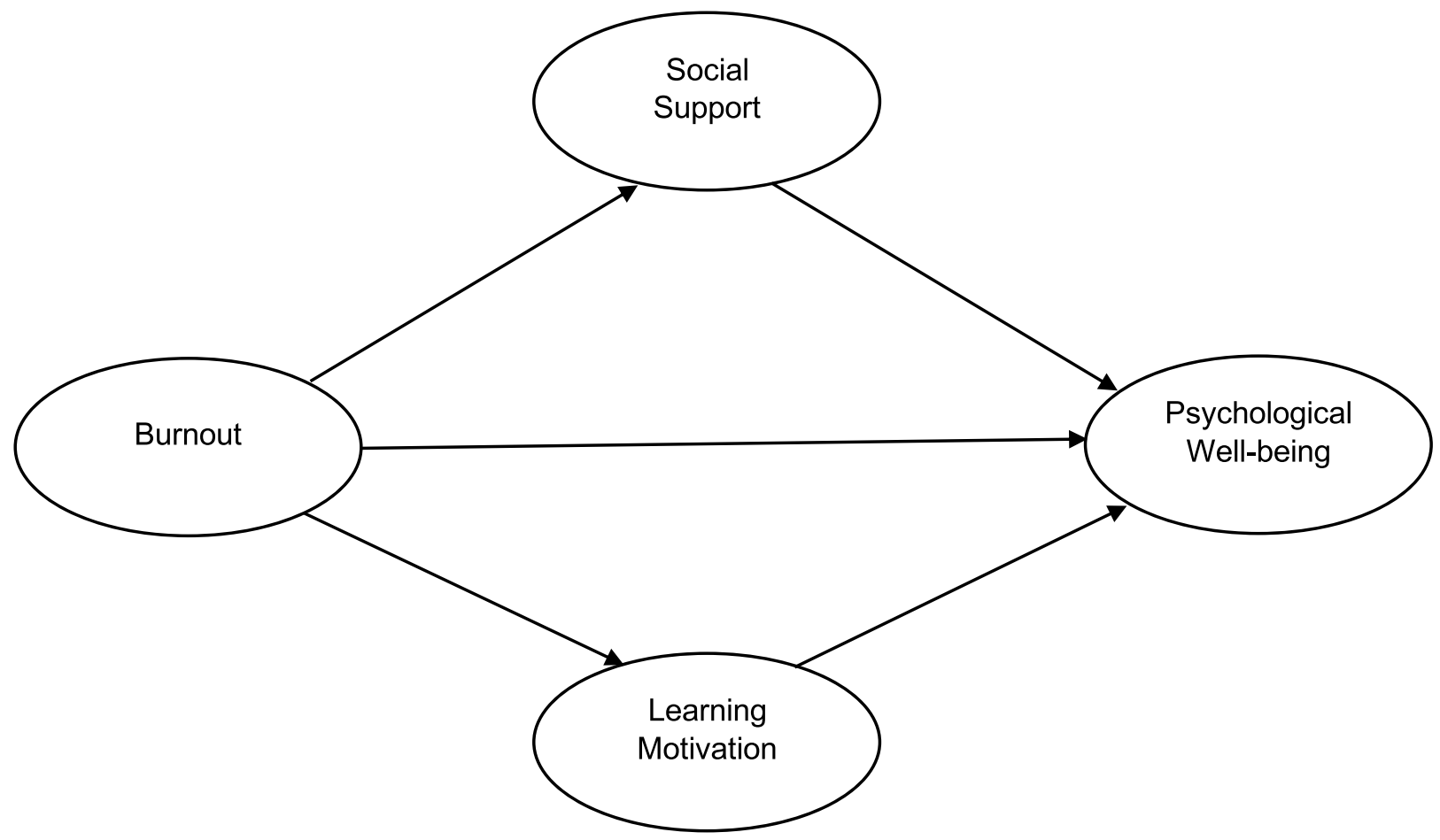

Figure I The suggested path mediation model showing mediation effect of social support and learning motivation in the link between burnout and psychological well-being. 
Table 3 Standardized Indirect Effects and 95\% Confidence Intervals

\begin{tabular}{|l|l|l|l|}
\hline \multirow{2}{*}{ Model Pathways } & \multirow{2}{*}{ Estimated } & \multicolumn{2}{|l|}{$\mathbf{9 5 \% C l}$} \\
\cline { 3 - 4 } & & Lower & Upper \\
\hline $\mathrm{BRN} \rightarrow \mathrm{SS} \rightarrow \mathrm{PW}$ & 0.171 & 0.109 & 0.241 \\
$\mathrm{BRN} \rightarrow \mathrm{LMS} \rightarrow \mathrm{PW}$ & 0.092 & 0.047 & 0.144 \\
indEff $(\mathrm{SS})$ minus indef (LMS) & -0.078 & -0.013 & 0.165 \\
\hline
\end{tabular}

Note: Empirical $95 \% \mathrm{Cl}$ does not overlap with zero.

Abbreviations: Brn, burnout; SS, social support; PW, psychological well-being; LMS, learning motivation; indEff, indirect effect.

$\mathrm{CI}=0.109,0.241)$ is important for the relationship between burnout and well-being. Bootstrap CIs are listed in Table 3. Due to its social relevance (social support $=0.171$, 95\% $\mathrm{CI}=0.109-0.241)$ and learning motivation (learning motivation $=0.091,95 \% \mathrm{CI}=0.047-0.144)$, the indirect on burnout: psychological well-being was significant. Notably, the mediating effect of social support was as strong as that of learning motivation $(95 \% \mathrm{CI}=-0.013,0.165)$ and this was reported of the difference in the indirect effects. Indeed, the $95 \%$ confidence interval contains no null values, suggesting that social support and learning motivation have a significant indirect impact on burnout-psychological well-being relationship. Thus, our hypothesis chain mediation model (social support-learning motivation) was supported and fit chain mediation model was satisfactory (Figure 2).

\section{Discussion}

In this study, we examined the link between burnout and psychological well-being and the role of social support and learning motivation as mediators. Our study findings show that social support and learning motivation mediated the burnout and psychological well-being relationship. We also found that the study results supported those studies that showed social support such as perceived social support and student's well-being, such as social support reduced levels of stress and depressions among youth. ${ }^{15}$ Therefore, the model indicated that burnout could affect low psychological well-being through the chain mediation effect of social support and learning motivation.

Regarding our first hypothesis, the link between burnout and psychological well-being was confirmed by the sample of 486 university students with ages ranged between 18 and 35 years. Findings indicated that burnout as a syndrome has significant effects on both physical and psychological well-being. ${ }^{54}$ Therefore, results suggest that burnout was negatively linked with psychological wellbeing. Interestingly, social support, one of the independent

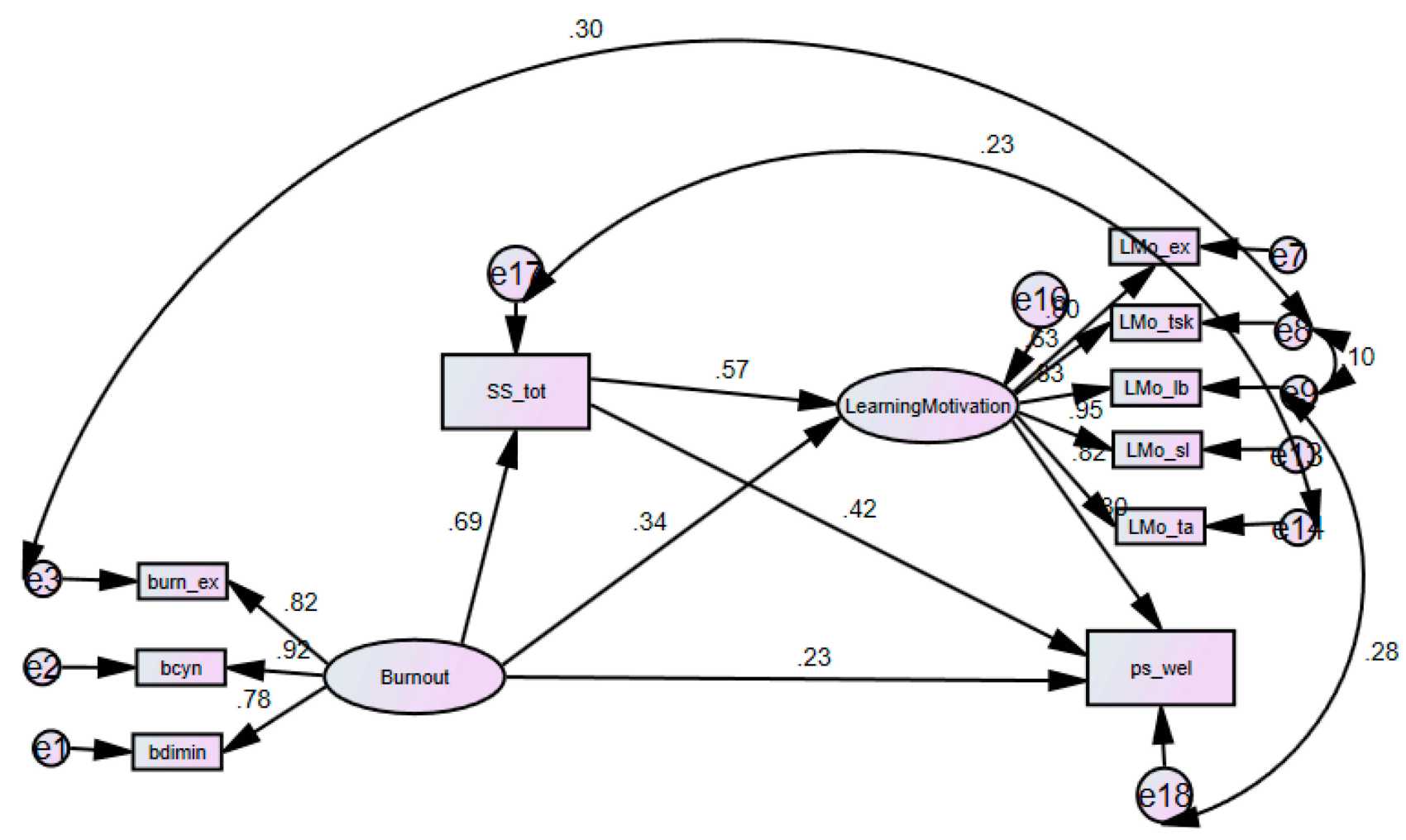

Figure 2 The final mediation model $(\mathrm{n}=486)$. Note factor loadings are standardized. Burn_ex, bcyn, and bdimin are three of burnout; social support, LMo_ex, LMo_tsk, LMo_Ib, LMo_sl, and LMo_ta are the sub scales of learning motivation and pswel is for psychological well-being; All the path coefficients are significant at $0.00 \mathrm{I}$ level. 
mediators, provided us with an empirical mediating role in the link between burnout and psychological well-being. In addition to this, it has been suggested that high social support may help to moderate the level of burnout and help to gain support from rich social networks that results in increased psychological well-being. ${ }^{15}$ Moreover, past literature has shown that the support gained from friends and family has negligible signs of burnout, $s$ as the level of burnout may decrease when an individual receives support from his/her colleagues or group members, for example, social support related with family and friends tend to lead to good achievements in life.

Our study also shows that the mediating role of social support was found to be consistent with the past studies among young Chinese adults, such as in collectivist cultures as seen in China, social support proved to be significantly important for young adults. For instance, Chinese adolescents positively predict academic success and better life satisfaction. ${ }^{29}$ Hence, the study findings support the notion that social support has a significant role in Asian cultures. Furthermore, literature also indicated that harmonious social links help in developing attachments with others. ${ }^{3}$

Regarding our third hypothesis, that the learning motivation mediated the link between burnout and psychological well-being was shown to be significant and findings support our model. The study findings highlight that learning motivation helps to increase the level of psychological well-being of an individual, such as learning environment and collaborative learning relationship and foster educational experience extend students' academic abilities, lead to enhanced motivation, and may help to overcome mental health related outcomes. ${ }^{55}$ Furthermore, consistent with the previous literature, findings indicate the role of social support and learning motivation affect the psychological well-being on one's life. Indeed, the motivation has positive implication for well-being in both individual as well as collectivistic cultures. ${ }^{30}$ Overall, findings support the hypothesis.

It has been noted that burnout has been considered one of the most influencing factors in the development of persistent stress and it may reduce the level of psychological wellbeing, for example, when students reach the point of extreme fatigue during their study period and they tend to act poorly in their studies with decreased levels of psychological well-being. Therefore, students who report an increase in motivation for learning tend to show lower burnout and better adjustment in their university life and report more meaningful life with increased psychological well-being. In addition, such students experience fewer psychological problems and attain higher grades in their academic studies. Therefore, study also suggests that at university level, first year students may report increased burnout and higher stress levels and this may continue from their transition period to their higher academic attainment with poor academic performance. ${ }^{56}$ Findings of the present research extend through indicating that social support and learning motivation may play independent contributions to the psychological well-being by using a multiple mediation model. In addition, findings indicate that burnout could account less for psychological well-being through the chain-mediating role of "social support-learning motivation" in Chinese culture.

Conversely, students with high social support and better learning motivation could perceive less burnout and thus experience better psychological well-being. Such findings could be due to the satisfactory social relationship and experience of high learning motivation and result in an increased level of psychological wellbeing. The study showed satisfactory consistent findings regarding social relationships and richer social support significantly improve the level of psychological wellbeing, ${ }^{57}$ particularly in collectivist cultures such as in Asian countries. ${ }^{58}$ Future study may be required to see the findings in various other cultures.

In sum, the present study attempts to examine the social support and learning motivation mediation model to uncover the link between burnout and psychological well-being. Findings provide support for pathway in the link: the role of social support and learning motivation as mediators that may help to provide an important contribution to the body of knowledge showing an increased psychological well-being in Chinese cultures. It suggests that designing such intervention programs may help to improve experiences with an opportunity to gain enhanced social support and better motivation for learning during their studies.

\section{Limitations and Suggestions}

It is also important to consider some study limitations associated with the present research while interpreting the results. First, we chose the sample for this current study from Chinese university students; therefore, the findings may not apply to the general population. Second, the measures used in the current study were based on selfreport and may have subjectivity. Therefore, multiple appraisal techniques such as longitudinal research designs 
and observational as well as experimental design may help in reducing such biases. The Ryff Scale of Psychological well-being has six domains that were not suggested as the study used psychological well-being as the total score. It is suggested that further study may explore the six domains of psychological well-being. Furthermore, concerning the measure of learning motivation, additional subscales from MSLQ, such as controlling the learning beliefs and goal orientation could also be included. Studies at university level need to recognize the importance of the role of learner affect and engagement in learning.

\section{Conclusion}

In conclusion, this study tried to determine that university students may value their psychological well-being as influenced by social support in terms of social support and learning motivation. Hence, findings of the present study have important implications for improving the students' level of psychological well-being. This finding shows the significance of social support and learning motivation to university students for their enhanced psychological well-being. Finally, the study reveals that burnout is closely associated to psychological well-being through social support and learning motivation, which has implications for students leading to their academic studies as well as for professionals related to the education sector.

\section{Acknowledgments}

The study was supported by the Department of Education Ministry of the People's Republic of China for Liberal Arts and Social Sciences Research (09YJCXLX014).

\section{Disclosure}

The authors report no conflicts of interest in this work.

\section{References}

1. Chang E, Lee A, Byeon E, Seong H, Lee SM. The mediating effect of motivational types in the relationship between perfectionism and academic burnout. Pers Individ Dif. 2016;89:202-210. doi:10.1016/j. paid.2015.10.010

2. Holt-Lunstad J, Smith TB, Layton JB, Brayne C. Social relationships and mortality risk: a meta-analytic review. PLoS Med. 2010;7(7): e1000316-e1000316. doi:10.1371/journal.pmed.1000316

3. Taylor SE, Welch WT, Kim HS, Sherman DK. Cultural differences in the impact of social support on psychological and biological stress responses. Psychol Sci. 2007;18(9):831-837. doi:10.1111/j.14679280.2007.01987.x

4. Shin H, Puig A, Lee J, Lee JH, Lee SM. Cultural validation of the maslach burnout inventory for Korean students. Asia Pac Educ Rev. 2011;12(4):633-639. doi:10.1007/s12564-011-9164-y
5. Schaufeli WB, Martinez IM, Pinto AM, Salanova M, Bakker AB. Burnout and engagement in university students: a cross-national study. J Cross Cult Psychol. 2002;33(5):464-481. doi:10.1177/ 0022022102033005003

6. Maslach C, Jackson SE. The measurement of experienced burnout. J Organ Behav. 1981;2(2):99-113. doi:10.1002/job.4030020205

7. Maslach C, Jackson S, Leiter M, Zalaquett C, Wood R. Evaluating Stress: A Book of Resources. USA: Scarecrow; 1997.

8. Klainin-Yobas P, Keawkerd O, Pumpuang W, Thunyadee C, Thanoi W, He HG. The mediating effects of coping on the stress and health relationships among nursing students: a structural equation modelling approach. $J$ Adv Nurs. 2014;70(6):1287-1298. doi:10. $1111 /$ jan. 12283

9. Eisenberg D, Gollust SE, Golberstein E, Hefner JL. Prevalence and correlates of depression, anxiety, and suicidality among university students. Am J Orthopsychiatry. 2007;77(4):534-542. doi:10.1037/ 0002-9432.77.4.534

10. Mikolajczyk RT, El Ansari W, Maxwell AE. Food consumption frequency and perceived stress and depressive symptoms among students in three European countries. Nutr J. 2009;8(1):31. doi:10.1186/1475-2891-8-31

11. Jun WH, Lee G. Comparing anger, anger expression, life stress and social support between Korean female nursing and general university students. J Adv Nurs. 2017;73(12):2914-2922. doi:10.1111/jan.13354

12. Stroebe W, Stroebe M, Abakoumkin G, Schut H. The role of loneliness and social support in adjustment to loss: a test of attachment versus stress theory. J Pers Soc Psychol. 1996;70(6):1241. doi:10.1037/0022-3514.70.6.1241

13. Gyasi RM, Yeboah AA, Mensah CM, Ouedraogo R, Addae EA. Neighborhood, social isolation and mental health outcome among older people in Ghana. J Affect Disord. 2019;259:154-163. doi:10.1016/j.jad.2019.08.024

14. Herberholz C, Phuntsho S. Social capital, outpatient care utilization and choice between different levels of health facilities in rural and urban areas of Bhutan. Soc Sci Med. 2018;211:102-113. doi:10.1016/ j.socscimed.2018.06.010

15. Koniarek J, Dudek B. Social support as a buffer in the stress-burnout relationship. Int J Stress Manag. 1996;3(2):99-106. doi:10.1007/ BF01857718

16. Bakker AB, Le Blanc PM, Schaufeli WB. Burnout contagion among intensive care nurses. $J$ Adv Nurs. 2005;51(3):276-287. doi:10.1111/ j.1365-2648.2005.03494.x

17. Asante KO. Social support and the psychological wellbeing of people living with HIV/AIDS in Ghana. Afr $J$ Psychiatry (Johannesbg). 2012;15(5):340-345. doi:10.4314/ajpsy.v15i5.42

18. Zhao J, Wang Y, Kong F. Exploring the mediation effect of social support and self-esteem on the relationship between humor style and life satisfaction in Chinese college students. Pers Individ Dif. 2014;64:126-130. doi:10.1016/j.paid.2014.02.026

19. Bruhn JG. The Sociology of Community Connections. Springer Science \& Business Media; 2011

20. Chen -W-W. The relations between perceived parenting styles and academic achievement in Hong Kong: the mediating role of students' goal orientations. Learn Individ Differ. 2015;37:48-54. doi:10.1016/j. lindif.2014.11.021

21. Eskin M. Self-reported assertiveness in Swedish and Turkish adolescents: a cross-cultural comparison. Scand J Psychol. 2003;44 (1):7-12. doi:10.1111/1467-9450.t01-1-00315

22. Osseiran-Waines N, Elmacian S. Types of social support: relation to stress and academic achievement among prospective teachers. Can J Behav Sci. 1994;26(1):1. doi:10.1037/0008-400X.26.1.1

23. Levpuscek MP, Zupancic M. The journal of early. J Early Adolesc. 2009;29(4):541-570.

24. Ryan RM, Deci EL. Self-determination theory and the facilitation of intrinsic motivation, social development, and well-being. $\mathrm{Am}$ Psychol. 2000;55(1):68. doi:10.1037/0003-066X.55.1.68 
25. Schunk D. Ch. 10. Classrooms and school influences» i schunk, Dale H. Paul R. Pintrich, Judith L. meece: motivation in education. Theor Res Appl. 2010;299-330.

26. Ratelle CF, Larose S, Guay F, Senécal C. Perceptions of parental involvement and support as predictors of college students' persistence in a science curriculum. J Fam Psychol. 2005;19(2):286. doi:10.1037/0893-3200.19.2.286

27. Deary IJ, Watson R, Hogston R. A longitudinal cohort study of burnout and attrition in nursing students. J Adv Nurs. 2003;43 (1):71-81. doi:10.1046/j.1365-2648.2003.02674.x

28. Yang H-J. Factors affecting student burnout and academic achievement in multiple enrollment programs in Taiwan's technical-vocational colleges. Int J Educ Dev. 2004;24(3):283-301. doi:10.1016/j. ijedudev.2003.12.001

29. Vansteenkiste M, Zhou M, Lens W, Soenens B. Experiences of autonomy and control among Chinese learners: vitalizing or immobilizing? J Educ Psychol. 2005;97(3):468. doi:10.1037/0022-0663.97.3.468

30. Chirkov V, Ryan RM, Kim Y, Kaplan U. Differentiating autonomy from individualism and independence: a self-determination theory perspective on internalization of cultural orientations and well-being. J Pers Soc Psychol. 2003;84(1):97. doi:10.1037/0022-3514.84.1.97

31. Baker JD. An investigation of relationships among instructor immediacy and affective and cognitive learning in the online classroom. Internet High Educ. 2004;7(1):1-13. doi:10.1016/j.iheduc.2003.11.006

32. Lee C-YS, Goldstein SE. Loneliness, stress, and social support in young adulthood: does the source of support matter? J Youth Adolesc. 2016;45(3):568-580. doi:10.1007/s10964-015-0395-9

33. Rostami Z, Abedi MR, Schaufeli WB, Ahmadi SA, Sadeghi AH. The Psychometric Characteristics of Maslach Burnout Inventory Student Survey: A Study Students of Isfahan University. 2014.

34. Pisarik CT. Motivational orientation and burnout among undergraduate college students. Coll Stud J. 2009;43(4):1238-1253.

35. Rostami Z, Abedi MR, Schaufeli W. Dose interest predicts academic burnout? Interdiscip J Contemp Res Bus. 2012.

36. Duncan TG, McKeachie WJ. The making of the motivated strategies for learning questionnaire. Educ Psychol. 2005;40(2):117-128. doi:10.1207/s15326985ep4002_6

37. Bong M. Academic motivation in self-efficacy, task value, achievement goal orientations, and attributional beliefs. J Educ Res. 2004;97 (6):287-298. doi:10.3200/JOER.97.6.287-298

38. McKenzie K, Gow K. Exploring the first year academic achievement of school leavers and mature-age students through structural equation modelling. Learn Individ Differ. 2004;14(2):107-123. doi:10.1016/j. lindif.2003.10.002

39. Wolters CA. Advancing achievement goal theory: using goal structures and goal orientations to predict students' motivation, cognition, and achievement. J Educ Psychol. 2004;96(2):236. doi:10.1037/ 0022-0663.96.2.236

40. Wilke RR. The effect of active learning on student characteristics in a human physiology course for nonmajors. Adv Physiol Educ. 2003;27(4):207-223. doi:10.1152/advan.00003.2002

41. Ryff CD, Keyes CLM. The structure of psychological well-being revisited. J Pers Soc Psychol. 1995;69(4):719. doi:10.1037/00223514.69.4.719

Psychology Research and Behavior Management

\section{Publish your work in this journal}

Psychology Research and Behavior Management is an international, peer-reviewed, open access journal focusing on the science of psychology and its application in behavior management to develop improved outcomes in the clinical, educational, sports and business arenas. Specific topics covered in the journal include: Neuroscience, memory and decision making; Behavior modification and management; Clinical
42. Andrews FM, Robinson JP. Measures of subjective well-being. Measures Personality Social Psychol Attitudes. 1991;1:61-114.

43. Tavakolizadeh J, Yadollahi H, Poorshafei H. The role of self regulated learning strategies in psychological well being condition of students. Procedia Soc Behav Sci. 2012;69:807-815. doi:10.1016/j. sbspro.2012.12.002

44. Dimitrijević AA, Marjanović ZJ, Dimitrijević A. Whichever intelligence makes you happy: the role of academic, emotional, and practical abilities in predicting psychological well-being. Pers Individ Dif. 2018;132:6-13. doi:10.1016/j.paid.2018.05.010

45. Springer KW, Hauser RM, Freese J. Bad news indeed for Ryff's sixfactor model of well-being. Soc Sci Res. 2006;35(4):1120-1131. doi:10.1016/j.ssresearch.2006.01.003

46. Zimet GD, Dahlem NW, Zimet SG, Farley GK. The multidimensional scale of perceived social support. J Pers Assess. 1988;52 (1):30-41. doi:10.1207/s15327752jpa5201_2

47. Wongpakaran T, Wongpakaran N, Ruktrakul R. Reliability and validity of the multidimensional scale of perceived social support (MSPSS): thai version. Clin Pract Epidemiol Mental Health. 2011;7 (1):161. doi: $10.2174 / 1745017901107010161$

48. Pampaka M, Hutcheson G, Williams J. Handling missing data: analysis of a challenging data set using multiple imputation. Int J Res Meth Educ. 2016;39(1):19-37. doi:10.1080/1743727X.2014.979146

49. Fang J, Wen Z, Zhang M, Sun P. The analyses of multiple mediation effects based on structural equation modeling. J Psychol Sci. 2014;37 (3):735-741.

50. Hu L, Bentler PM. Cutoff criteria for fit indexes in covariance structure analysis: conventional criteria versus new alternatives. Struct Equ Modeling. 1999;6(1):1-55. doi:10.1080/10705519909540118

51. Hooper D, Coughlan J, Mullen MR. Structural equation modelling: guidelines for determining model fit. Electron $J$ Bus Res Methods. 2008;6(1):53-60.

52. Çokluk Ö, Şekercioğlu G, Büyüköztürk Ş. Sosyal bilimler için çok değişkenli istatistik: SPSS ve LISREL uygulamaları. Pegem Atif Indeksi. 2018;001-414.

53. MacKinnon DP, Lockwood CM, Williams J. Confidence limits for the indirect effect: distribution of the product and resampling methods. Multivariate Behav Res. 2004;39(1):99-128. doi:10.1207/ s15327906mbr3901 4

54. Shin H, Noh H, Jang Y, Park YM, Lee SM. A longitudinal examination of the relationship between teacher burnout and depression. J Employ Couns. 2013;50(3):124-137. doi:10.1002/j.2161-1920.2013.00031.x

55. Zepke N, Leach L. Improving student engagement: ten proposals for action. Active Learn High Educ. 2010;11(3):167-177. doi:10.1177/ 1469787410379680

56. Bernhard C. A survey of burnout among college music majors: a replication. Music Perform Res. 2010;3.

57. Zhu H. Social support and affect balance mediate the association between forgiveness and life satisfaction. Soc Indic Res. 2015;124 (2):671-681. doi:10.1007/s11205-014-0790-8

58. Cuesta-Zamora C, González-Martí I, García-López LM. The role of trait emotional intelligence in body dissatisfaction and eating disorder symptoms in preadolescents and adolescents. Pers Individ Dif. 2018;126:1-6. doi:10.1016/j.paid.2017.12.021

applications; Business and sports performance management; Social and developmental studies; Animal studies. The manuscript management system is completely online and includes a very quick and fair peer-review system, which is all easy to use. Visit http://www. dovepress.com/testimonials.php to read real quotes from published authors. 